\title{
Phylogeny and ontogeny of the habenular structure
}

\author{
Hidenori Aizawa ${ }^{1,2}$, Ryunosuke Amo ${ }^{2,3}$ and Hitoshi Okamoto ${ }^{2,3,4}$ \\ Department of Molecular Neuroscience, Medical Research Institute, Tokyo Medical and Dental University, Tokyo, Japan \\ 2 Laboratory for Developmental Gene Regulation, RIKEN Brain Science Institute, Saitama, Japan \\ ${ }^{3}$ Department of Life Science and Medical Bioscience, Graduate School of Advanced Science and Engineering, Waseda University, Tokyo, Japan \\ ${ }^{4}$ Core Research for Evolutional Science and Technology, Japan Science and Technology Agency, Tokyo, Japan
}

\section{Edited by:}

Tomomi Shimogori, RIKEN Brain

Science Institute, Japan

Reviewed by:

Jozsef Z. Kiss, University of Geneva,

Switzerland

Gregor Kasprian, Medical University

Vienna, Austria

*Correspondence:

Hidenori Aizawa, Department of Molecular Neuroscience, Medical Research Institute, Tokyo Medical and Dental University, 1-5-45 Yushima, Bunkyzo-ku, Tokyo 113-8510, Japan. e-mail: haizawa.aud@mri.tmd.ac.jp

\begin{abstract}
Habenula is an epithalamic nucleus connecting the forebrain with the ventral midbrain and hindbrain that plays a pivotal role in decision making by regulating dopaminergic and serotonergic activities. Intriguingly, habenula has also attracted interest as a model for brain asymmetry, since many vertebrates show left-right differences in habenula size and neural circuitry. Despite the functional significance of this nucleus, few studies have addressed the molecular mechanisms underlying habenular development. Mammalian habenula consists of the medial and lateral habenulae, which have distinct neural connectivity. The habenula shows phylogenetic conservation from fish to human, and studies using genetically accessible model animals have provided molecular insights into the developmental mechanisms of the habenula. The results suggest that development of the habenular asymmetry is mediated by differential regulation of the neurogenetic period for generating specific neuronal subtypes. Since the orientation and size ratio of the medial and lateral habenulae differ across species, the evolution of those subregions within the habenula may also reflect changes in neurogenesis duration for each habenular subdivision according to the evolutionary process.
\end{abstract}

Keywords: habenula, brain asymmetry, lateralization, monoamines, neurogenesis, interpeduncular nucleus, zebrafish, evolution
The habenula in the epithalamus connects the limbic forebrain with the midbrain and hindbrain where the monoaminergic neurons are concentrated. This nucleus has attracted growing interest because recent studies implicated it as a negative source of the reward signal in midbrain dopaminergic neurons (Hikosaka, 2010), and it plays critical roles in the pathophysiology of psychiatric disorders including depression (Lecourtier et al., 2004; Roiser et al., 2009; Sartorius et al., 2010; Li et al., 2011).

The habenular structure is well conserved across species, with all vertebrates examined possessing the efferent pathway of the habenula, which is called the fasciculus retroflexus or habenulointerpeduncular tract which runs longitudinally from the epithalamus to the ventral midbrain (Concha and Wilson, 2001). This conservation allowed us to use model animals that are more amenable to genetic manipulation to analyze the development and function of the habenular circuitry (Okamoto et al., 2011). The habenula is also peculiar in that many vertebrates show conspicuous left-right asymmetry in its size and cytoarchitecture (Concha and Wilson, 2001), suggesting this brain region as a good model for the analysis of brain asymmetry.

Herein we discuss this expanding research field on the habenula and summarize recent findings from the genetic analysis of animal models like zebrafish.

\section{HABENULA MODULATES ANIMAL BEHAVIORS VIA MONOAMINERGIC REGULATION}

Habenula was previously implicated as a regulatory center for the dopaminergic and serotonergic systems in the central nervous system (CNS), based on studies showing that lesions in the habenula led to increased monoamine metabolism (Nishikawa and Scatton, 1985; Nishikawa et al., 1986). In addition, electric stimulation of the habenula inhibited the firing of dopaminergic and serotonergic neurons in anesthetized animals (Wang and Aghajanian, 1977; Christoph et al., 1986). However, it has remained unclear when the habenular neurons are activated in the behaving animals. Recent electrophysiological studies in monkeys also revealed activation of the lateral habenular neurons in response to the aversive stimuli and outcomes that seem inappropriate for the chosen behaviors, as opposed to the inactivation of midbrain dopaminergic neurons (Matsumoto and Hikosaka, 2007, 2009). These results substantiated the view that the habenula act as a negative source for the monoaminergic systems.

Based on these previous observations, lateral habenula was implicated in the pathophysiology of psychiatric disorders such as schizophrenia and depression in which dysregulation of the monoaminergic systems has long been postulated as the neural bases of these diseases (Sandyk, 1991; Ellison, 1994). Depressive patients show increased cerebral blood flow in the habenula (Morris et al., 1999; Roiser et al., 2009), and excitatory synapses onto the lateral habenular neurons are potentiated in learned helpless rats showing depression-like behaviors (Li et al., 2011). It was therefore hypothesized that increased excitability in the lateral habenula may underlie the pathophysiology of depression. Thus, reducing hyperexcitability in the habenula by surgical methods such as deep brain stimulation is a plausible future therapy strategy for drug-resistant depression (Sartorius et al., 2010). 
However, only a limited number of studies have addressed the function of the medial habenula, probably because this structure is too small to be targeted by conventional experimental methods such as lesioning or stimulation. Genetic manipulation of the medial habenular neurons and their homologs confirmed that medial habenula is essential in controlling nicotine intake (Fowler et al., 2011) and fear expression (Agetsuma et al., 2010).

Despite this demonstrated functional significance of habenula, examining the function, and developmental mechanism for a subset of functional pathways remained difficult, mainly because there was no model animal with neural circuitry that is readily amenable to a genetic approach.

\section{PHYLOGENETIC CONSERVATION OF THE MEDIAL AND LATERAL HABENULAR PATHWAYS}

The mammalian habenula is subdivided into medial and lateral habenulae, each of which project to different targets. The medial habenula projects, almost exclusively, to the interpeduncular nucleus (IPN; Herkenham and Nauta, 1979), whereas the lateral habenula projects to a variety of nuclei such as rostromedial tegmental nucleus (Herkenham and Nauta, 1979; Jhou et al., 2009; Kaufling et al., 2009), raphe nuclei, substantia nigra, ventral tegmental area (Herkenham and Nauta, 1979), and the nucleus incertus (Goto et al., 2001; Olucha-Bordonau et al., 2003; Figure 1A). However, few studies have addressed whether subregions homologous to the medial and lateral habenulae in mammals are present in the other vertebrates, despite the fiber bundle

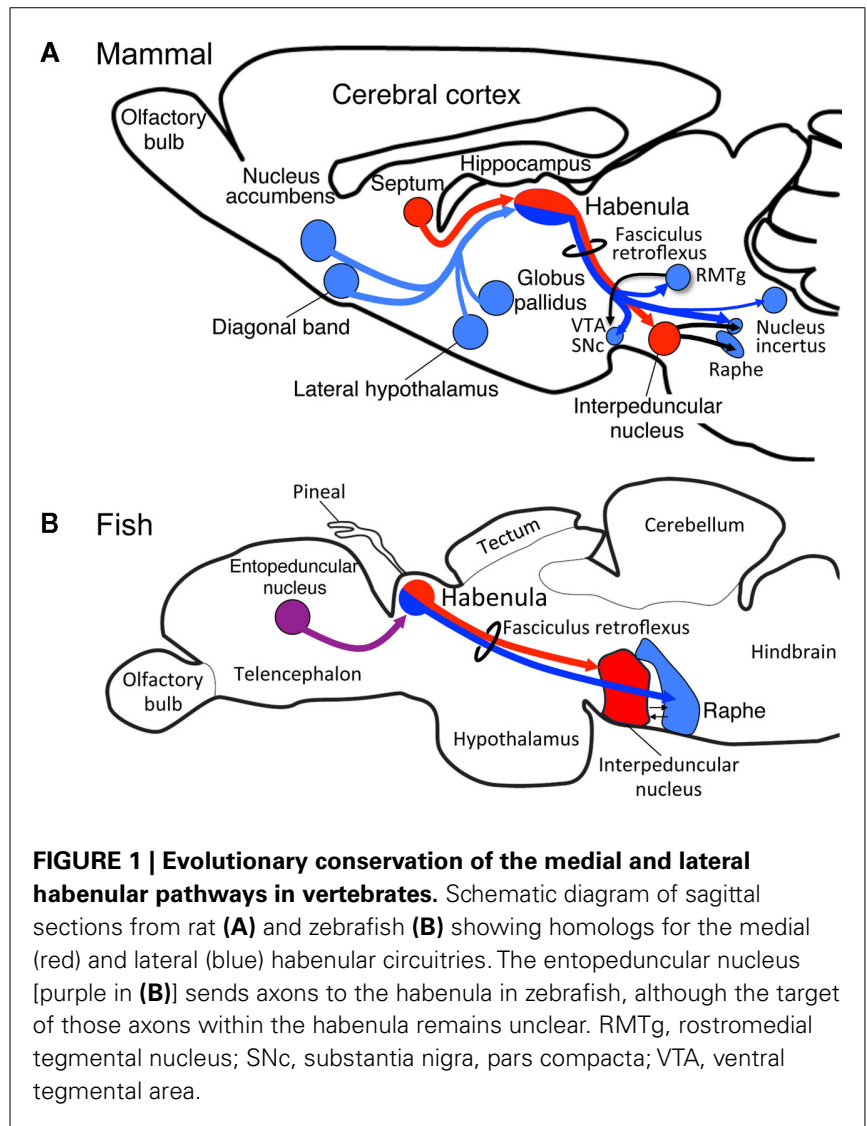

called the fasciculus retroflexus connecting the habenula with the ventral midbrain in all vertebrates examined (Figures 1A,B).

Subdivision of the habenula into the medial and lateral habenulae occurs in reptiles, birds, and mammals (Figures 2A-C; Concha and Wilson, 2001). In lizard, the mediolateral organization of the habenula and its efferent connectivity is comparable to that found in mammals, in which the medial and lateral habenulae project to the IPN and raphe nuclei, respectively (Distel and Ebbesson, 1981; Engbretson et al., 1981). Although the medial habenula homolog that projects to the IPN is conserved from fish to mammals (red lines in Figures 1A,B), it remains unclear whether fish and amphibia have lateral habenular pathways. Fish and amphibian habenulae can be subdivided into dorsal and ventral habenulae based on the cytoarchitecture (Figures 2D,E; Braford and Northcutt, 1983; Kemali and Lazar, 1985), with some habenular neurons projecting to the serotonergic neuron-rich raphe nuclei, although studies showing this lacked detailed positional descriptions of such neurons with respect to the dorsal and ventral organization of the habenula (Kemali and Lazar, 1985; Yanez and Anadon, 1996; Guglielmotti and Fiorino, 1998; Tomizawa et al., 2001; Aizawa et al., 2005; Bianco et al., 2008).

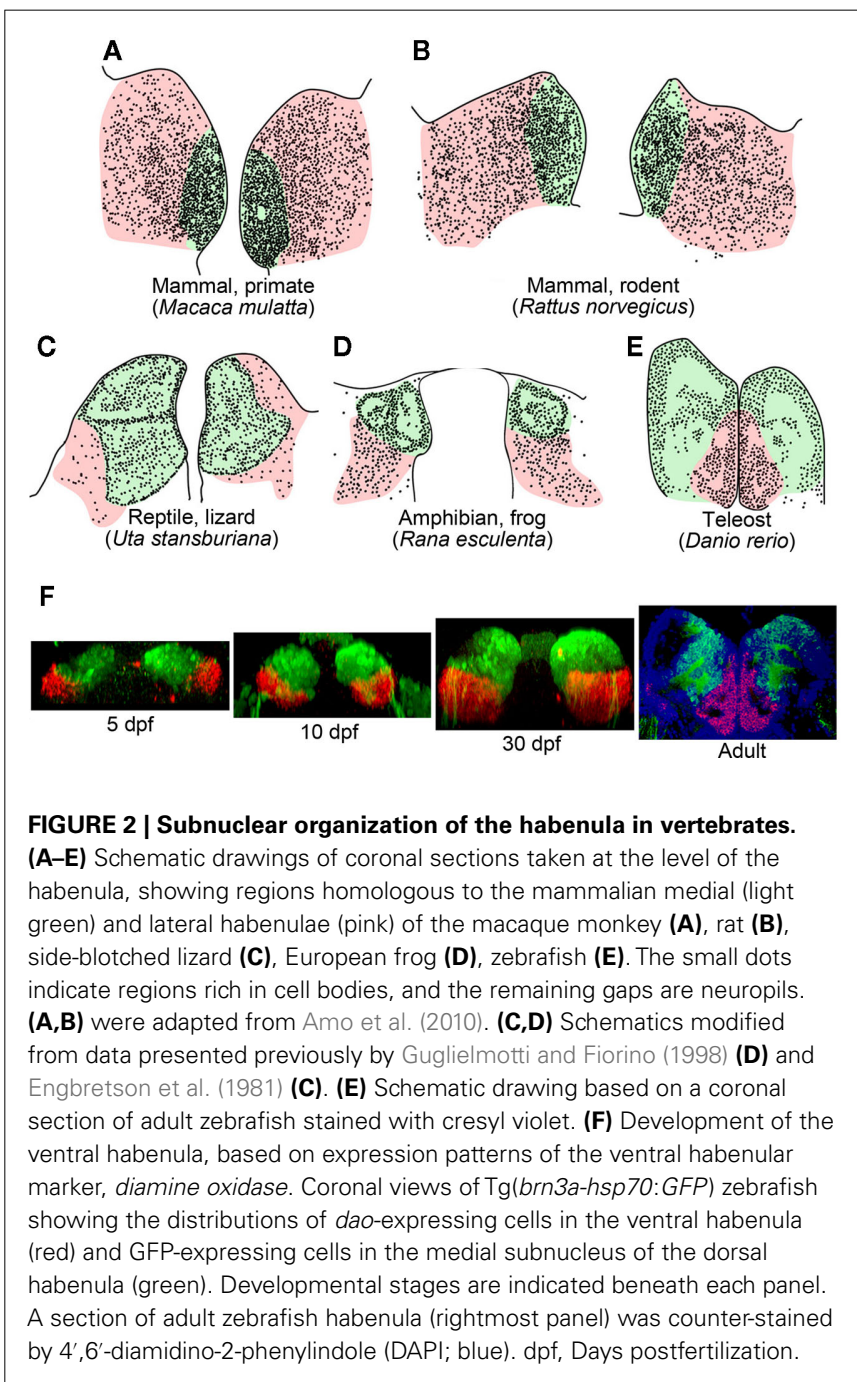


Two possibilities may be considered to explain the evolution of the medial and lateral pathways in the habenula. One is that the lateral habenular pathway was newly added to the habenula, which originally projected only to IPN. Another possibility assumes the presence of both the medial and lateral habenula homologs in the lower vertebrates as in mammals, and expansion or shrinkage of the lateral habenula homolog later occurring in response to changing environmental demands on the brain. To address these possible explanations, we examined the level of medial and lateral habenulae conservation in zebrafish, a model that is genetically accessible and amenable for live imaging. Analysis of transgenic fish revealed that the dorsal habenula projects to the IPN and is thus homologous to the medial habenula of mammals (red lines in Figures 1A,B; Aizawa et al., 2005). In contrast, zebrafish ventral habenula sends axons to the median raphe (blue line in Figure 1B; Amo et al., 2010) and expresses protocadherin 10a (Hirano et al., 1999), a specific marker of the lateral habenula of mammals. Thus, the fish ventral habenula is homologous to the lateral habenula of mammals. These findings confirmed that the medial and lateral habenular pathways are well conserved between fish and mammals.

Since the ventral habenula is located ventromedially to the dorsal habenula in adult zebrafish brain (Figure 2E), the orientations of these structures appear opposite to that of the mammalian habenula in the mediolateral direction (Figures 2A,B; Amo et al., 2010). In contrast, the lateral habenula in macaque monkeys is expanded dorsally (pink in Figure 2A), whereas the medial habenula is pushed ventromedially and appears to be residual (light green in Figure 2A; Mikula et al., 2007). Gene expression analyses revealed that the ventromedially positioned ventral habenula (red in Figure 2F) in the adult zebrafish originated from the region of primordium lateral to the dorsal habenula (green in Figure 2F), and it then gradually migrated ventromedially as development progressed (Amo et al., 2010). This suggested that zebrafish habenulae emerge during development with mediolateral orientation similar to that of the mammalian medial and lateral habenulae.

The differential size ratio of the medial and lateral habenulae across species may reflect the extent of the cortical input through the basal ganglia to the lateral habenula (Yanez and Anadon, 1996). Since the number of neurons in the medial and lateral habenulae is determined by developmental processes such as proliferation, differentiation, and cell death, the evolutionary changes in subnuclear organization in the habenula may reflect species-specific modulation of the neurogenetic processes (Finlay and Darlington, 1995). Thus, enlargement of the lateral habenula by increasing the duration of neurogenesis might result in finer tuning of the monoaminergic systems to evaluate a larger amount of sensory information.

\section{MOLECULAR MECHANISM FOR THE AXONAL GUIDANCE OF THE HABENULAR CIRCUITRY}

Epithalamus including habenular primordium in mammals is in prosomere 2 domain (p2; Figure $3 \mathbf{A}$ ). A recent study revealed that Fgf8 expressed in the dorsal diencephalon including p2 (light blue in Figure 3A) was indispensable for development of the habenula,

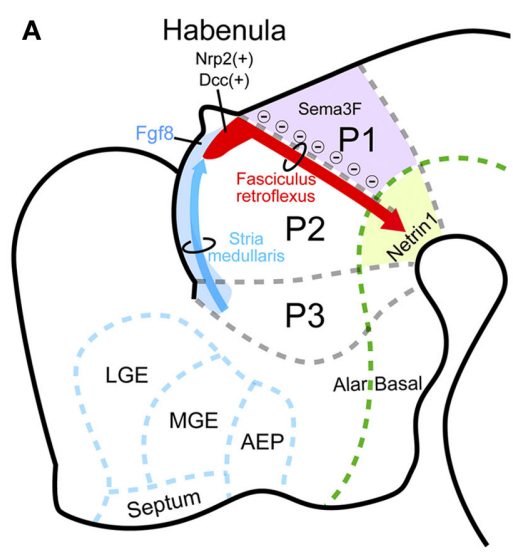

FIGURE 3 | Developmental mechanism of the habenula in genetically accessible model animals. (A) Schematic diagram of a sagittal section from E14 rat showing the habenular primordium (red), developing axons from the habenula (fasciculus retroflexus, red arrow) extending in between prosomere $1(\mathrm{p} 1)$ and prosomere 2 (p2), and developing afferents to the habenula (stria medullaris) running along the dorsolateral surface of prosomere 3 (p3) and p2 (as blue arrow). The dorsal part of p2 and p3 expressed Fgf8 (light blue), which drives essential signaling for habenular development. Areas expressing guidance molecules for the habenular axons are designated by purple (Sema3F) and light green (Netrin1). AEP, anterior entopeduncular area; LGE, lateral ganglionic eminence; MGE, medial ganglionic eminence. The rostral and dorsal side is oriented the left and top of the panel, respectively. (B) Neurogenetic model for the generation of left-right differences in the zebrafish dorsal habenula. At

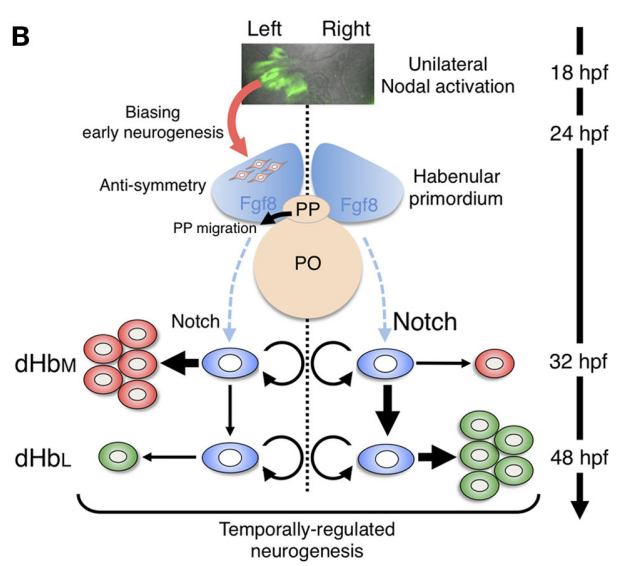

18-24 h postfertilization (hpf), Nodal, and its downstream genes (green in the top panel showing dorsal view of Tg[lefty 1:GFP]) are activated in the left dorsal diencephalon. Unilateral activation of Nodal signaling in the dorsal diencephalon directs the parapineal migration toward left (black arrow in the middle panel) and biases early neurogenesis in the habenular primordium with anti-symmetric nature in the presence of Fgf8 (red arrow between top and middle panels). Under those influences, common neural stem cells (light blue in the bottom panel) in the habenular primordium start to generate neural precursors for the lateral subnucleus $(\mathrm{dHbl}$, red in the bottom panel) predominantly on the left side, under the influence of reduced Notch activity, and then neural precursors for the medial subnucleus ( $\mathrm{dHbM}$, green in the bottom panel) predominantly on the right side during late stages of neurogenesis peaked at $48 \mathrm{hpf}$. PO, pineal organ; PP, parapineal organ. 
since mutants with reduced $F g f 8$ showed a dose-dependent reduction in habenula size (Martinez-Ferre and Martinez, 2009). Neuronal precursors for the habenular neurons are associated with the neuroepithelial domain expressing transcription factor $D b x 1$ in the dorsal part of p2 (Vue et al., 2007; Quina et al., 2009). That region first starts to generate lateral habenular precursors (E13 in rat), followed by the medial habenular precursors (E15 in rat) showing the lateromedial gradient in neurogenesis (Angevine, 1970; Altman and Bayer, 1979). Initial axonal growth from the habenular nucleus was observed as early as E13 in rats (Funato et al., 2000), and bundles of the efferent projection (fasciculus retroflexus) runs ventrocaudally between the $\mathrm{p} 1$ and $\mathrm{p} 2$ domains to form the segmental scaffold in the embryonic diencephalon (red arrow in Figure 3A; Figdor and Stern, 1993; Funato et al., 2000). Domain p1 expressing semaphorin 3F (Sema3F; purple in Figure 3A), which utilizes neuropilin-2 (Nrp2) as a receptor, showed repulsive effects on the Nrp2-expressing axons from the habenula in vitro (red in Figure 3A; Funato et al., 2000). Interaction between Sema3F and Nrp2 is needed in the formation of fasciculated habenular axons (fasciculus retroflexus), since mice lacking Sema3F or Nrp2 showed defasciculation or absence of this axonal bundle, respectively (Chen et al., 2000; Sahay et al., 2003). In addition to that repulsive guidance, the habenular axons expressing Deleted in colorectal cancer (Dcc; red in Figure 3A) are also guided by the attractive signal, Netrin1 (light green in Figure 3A; ligand for Dcc receptor), in the floor plate (Funato et al., 2000). Despite this accumulating evidence for the mechanisms guiding habenular axons, it remains unclear how and when the afferents to the habenula (stria medullaris; blue arrow in Figure 3A) develop. Molecular analysis of transgenic animals expressing fluorescent proteins in the habenular afferents or efferents is therefore an important way to decipher the mechanism driving habenular circuitry emergence.

\section{HABENULA AS A MODEL SYSTEM FOR ANALYZING LEFT-RIGHT ASYMMETRY IN THE BRAIN}

Brain asymmetry developed as a basic feature of the CNS across the vertebrates (Toga and Thompson, 2003). This feature is considered advantageous for efficient processing of neural information, such that each hemisphere is engaged in the differential regulation of behavior (Vallortigara and Rogers, 2005). However, few studies have addressed the molecular mechanism behind the generation of asymmetric brain, because there is no model animal in which neural development in both hemispheres is readily amenable to a genetic approach.

The region homologous to the medial habenula and the other epithalamic structures such as the parapineal/parietal organ show conspicuous left-right differences in many vertebrates including fish (Concha and Wilson, 2001). For instance, the left habenula has larger neuropil, and the parapineal organ innervating the left habenula is found only on the left side. Asymmetry in the habenula is observed not only in size and cytoarchitecture, but also in the axonal projection pattern to IPN (Aizawa et al., 2005; Gamse et al., 2005). Among the animals with asymmetry in the habenula, zebrafish are genetically accessible and amenable to imaging of morphogenetic processes because of their transparency during development. Furthermore, the availability of markers such as kctd12 family genes, many of which are expressed asymmetrically in the zebrafish habenula (Gamse et al., 2003), enabled us to detect the consequent changes of the brain asymmetry in the mutant animals. Thus the habenular asymmetry in zebrafish provides an opportunity to examine the molecular mechanisms underlying the development of brain asymmetry.

\section{DEVELOPMENTAL MECHANISM THAT DIRECTS THE EPITHALAMIC ASYMMETRY}

Two possible developmental mechanisms have been suggested to drive epithalamic asymmetry; determination of the asymmetry direction, and generation of left-right difference. Prior to a series of key discoveries in this field, the first clue came from studies showing correlation between the direction of Nodal signal activation and asymmetry in the epithalamic region (Concha et al., 2000; Liang et al., 2000). Nodal genes are expressed in the left lateral plate mesoderm (LPM) and play a central role in asymmetric formation of the visceral organ in all vertebrates examined so far (Hamada et al., 2002). In fish, a Nodal-related gene (cyclops) is unilaterally activated in the left dorsal diencephalon at $18-20 \mathrm{~h}$ postfertilization (hpf; top panel in Figure 3B; Rebagliati et al., 1998; Sampath et al., 1998). Zebrafish mutant analyses also revealed that the direction of habenular asymmetry (laterality) and position of the parapineal organ were randomly determined when Nodal signaling in the diencephalon was absent or activated bilaterally (Concha et al., 2000). Indeed, live imaging of Nodal activation in the embryonic diencephalon confirmed that the direction of Nodal activation was concordant with that of the habenular laterality (Aizawa et al., 2005). This lateralized activation is reportedly dependent on the activity of Nodal-related gene southpaw, which emanates from the left LPM (Long et al., 2003). However, there could also be a brain-specific mechanism underlying the asymmetric Nodal activation, based on studies showing symmetric Nodal activation in the embryonic diencephalon despite intact Nodal expression only in the left LPM in mutants defective in either Six3 or Wnt/Axin1/ $\beta$-catenin (Carl et al., 2007; Inbal et al., 2007). Following the transient activation of Nodal signaling on the left side, the primordial parapineal organ migrates from the midline toward the left side at $28-36 \mathrm{hpf}$ (black arrow in the middle panel of Figure 3B; Concha et al., 2003). Laser ablation of the premigratory parapineal organ and mutants defective in parapineal migration both show symmetric habenula (Concha et al., 2003; Gamse et al., 2003; Snelson et al., 2008), suggesting that interaction between the parapineal and habenular anlage ensures elaboration of the lateralized epithalamus.

\section{NEUROGENETIC ASYMMETRY DETERMINING CELL NUMBER IN THE LEFT AND RIGHT HABENULAE}

Despite the pivotal role that Nodal signaling plays in determining the direction of habenular asymmetry during the early stages of development, a size difference is still apparent between hemispheres, even in mutants that lacked Nodal activation in the developing dorsal diencephalon (Concha et al., 2000). This suggested that Nodal signaling might be dispensable for the emergence of left-right differences in habenula size.

The developing epithalamus in zebrafish seems to display $f g f 8$ dependent anti-symmetry (asymmetry without a directional bias) 
in nature that is independent of Nodal signaling (middle panel of Figure 3B). This was shown in acerebellar zebrafish mutant embryos, which showed reduced $f g f 8$ function and symmetric habenula on both sides and a parapineal organ at the midline (Regan et al., 2009). A recent study also revealed that pharmacological inhibition of Nodal signaling disrupts the early asymmetric neurogenesis in habenula and parapineal migration, indicating that unilateral activation of Nodal signaling could act on the $f g f 8$ expressing anti-symmetric domain in the embryonic epithalamus to bias both the habenular neurogenesis and parapineal migration toward the left side during early development (middle panel of Figure 3B; Roussigne et al., 2009).

Left-right difference in the number of neurons that belong to each subnucleus underlies the development of habenular asymmetry. The issue of habenular asymmetry is based on the mechanism by which these subnuclei are asymmetrically generated during development, since the dorsal habenula in zebrafish consists of medial ( $\mathrm{dHbM}$, green in the right most panel of Figure $2 \mathrm{~F}$ ) and lateral subnuclei ( $\mathrm{dHb}$, blue region dorsolateral to $\mathrm{dHbм}$ in the right most panel of Figure 2F), each of which has a distinct projection pattern to the IPN (Aizawa et al., 2005; Gamse et al., 2005). The birthdate analyses by bromodeoxyuridine-pulse labeling experiments indicated that neural precursors for the $\mathrm{dHbL}$ (red in the bottom panel of Figure 3B) emerge at an earlier stage than those for the $\mathrm{dHbM}$ (green in the bottom panel of Figure 3B). In addition, more neural precursors are born on the left side than on the right side, most likely due to the right-dominant asymmetry in the activity of Notch signaling, which suppresses neurogenetic activity in neural stem cells (Louvi and Artavanis-Tsakonas, 2006). Since both the neural precursors for the $\mathrm{dHbL}$ and the $\mathrm{dHbM}$ are derived from common stem cells (blue in the bottom panel of Figure 3B; Aizawa et al., 2007), more neurogenesis in the left habenula induces quicker depletion of the stem cells in the left habenula than in the right habenula. This causes more neurogenesis from the remaining stem cells on the right side to give rise to more $\mathrm{dHbm}$ neurons

\section{REFERENCES}

Agetsuma, M., Aizawa, H., Aoki, T., Nakayama, R., Takahoko, M., Goto, M., Sassa, T., Amo, R., Shiraki, T., Kawakami, K., Hosoya, T., Higashijima, S., and Okamoto, $\mathrm{H}$. (2010). The habenula is crucial for experience-dependent modification of fear responses in zebrafish. Nat. Neurosci. 13, 1354-1356.

Aizawa, H., Bianco, I. H., Hamaoka, T., Miyashita, T., Uemura, O., Concha, M. L., Russell, C., Wilson, S. W., and Okamoto, H. (2005). Laterotopic representation of left-right information onto the dorso-ventral axis of a zebrafish midbrain target nucleus. Curr. Biol. 15, 238-243.

Aizawa, H., Goto, M., Sato, T., and Okamoto, H. (2007). Temporally regulated asymmetric neurogenesis causes left-right difference in the zebrafish habenular structures. Dev. Cell 12, 87-98.
Altman, J., and Bayer, S. A. (1979). Development of the diencephalon in the rat. V. Thymidine-radiographic observations on internuclear and intranuclear gradients in the thalamus. J. Comp. Neurol. 188, 473-499.

Amo, R., Aizawa, H., Takahoko, M., Kobayashi, M., Takahashi, R., Aoki, T., and Okamoto, H. (2010). Identification of the zebrafish ventral habenula as a homolog of the mammalian lateral habenula. J. Neurosci. 30, 1566-1574.

Angevine, J. B. Jr. (1970). Time of neuron origin in the diencephalon of the mouse. An autoradiographic study. J. Comp. Neurol. 139, 129-187.

Bianco, I. H., Carl, M., Russell, C., Clarke, J. D., and Wilson, S. W. at the level of axon terminal morphology. Neural Dev. 3, 9. (2008). Brain asymmetry is encoded on the right side than on the left side at later stages of embryonic development, which ultimately leads to the left-right asymmetric subnuclear organization of the dorsal habenula (Aizawa et al., 2007).

These studies together suggest that lateralization may work by regulating the reciprocal expansion or contraction of subregions within a particular brain region rather than by specifying unique structures on either left or right sides. This allows for variation of the brain structure between species, dependent upon the extent of lateralization, without the need for a complete re-definition of the structure in lateralized and non-lateralized species.

\section{PERSPECTIVES}

Recent evidence supports the view that modification of the duration of neurogenesis, during which the particular neuronal subtype is generated, can cause expansion or shrinkage of the particular habenula subdivision. This may in turn lead to a species-specific subnuclear organization of brain asymmetry and enable animals to adjust the subset predominance of the habenular circuitry according to the prevailing evolutionary adaptation. Further analysis of the molecular mechanisms that determine the number of neurons in each subregion of the habenula by using genetically accessible animals will be indispensable to understand how the habenula evolved in vertebrates.

\section{ACKNOWLEDGMENTS}

We thank the members of our laboratories for valuable discussions related to this work. This research was supported by a Grant-in-Aid for Scientific Research for Young Scientists (B) (KAKENHI21700370) from the Ministry of Education, Culture, Sports, Science and Technology (MEXT) to Hidenori Aizawa, and from RIKEN BSI and Core Research for Evolutional Science and Technology of Japan Science and Technology Agency to Hitoshi Okamoto. Ryunosuke Amo is a recipient of a fellowship of the Japan Society for the Promotion of Science for Junior Scientists.
Braford, M. R. J., and Northcutt, R. G. (1983). "Organization of the diencephalon and pretectum of rayfinned fishes," in Fish Neurobiology. 2. Higher Brain Areas and Functions, eds R. E. Davis and R. G. Northcutt (Ann Arbor: University of Michigan Press), 117-163.

Carl, M., Bianco, I. H., Bajoghli, B. Aghaallaei, N., Czerny, T., and Wilson, S. W. (2007). Wnt/Axin1/betacatenin signaling regulates asymmetric nodal activation, elaboration, and concordance of CNS asymmetries. Neuron 55, 393-405.

Chen, H., Bagri, A., Zupicich, J. A., Zou, Y., Stoeckli, E., Pleasure, S. J., Lowenstein, D. H., Skarnes, W. C., Chedotal, A., and Tessier-Lavigne, M. (2000). Neuropilin-2 regulates the development of selective cranial and sensory nerves and hippocampal mossy fiber projections. Neuron 25, 43-56.
Christoph, G., Leonzio, R., and Wilcox, K. (1986). Stimulation of the lateral habenula inhibits dopaminecontaining neurons in the substantia nigra and ventral tegmental area of the rat. J. Neurosci. 6, 613-619.

Concha, M. L., Burdine, R. D., Russell, C., Schier, A. F., and Wilson, S. W. (2000). A nodal signaling pathway regulates the laterality of neuroanatomical asymmetries in the zebrafish forebrain. Neuron 28, 399-409.

Concha, M. L., Russell, C., Regan, J. C., Tawk, M., Sidi, S., Gilmour, D. T., Kapsimali, M., Sumoy, L., Goldstone, K., Amaya, E., Kimelman, D., Nicolson, T., Grunder, S., Gomperts, M., Clarke, J. D., and Wilson, S. W. (2003). Local tissue interactions across the dorsal midline of the forebrain establish CNS laterality. $\mathrm{Neu}$ ron $39,423-438$. 
Concha, M. L., and Wilson, S. W. (2001). Asymmetry in the epithalamus of vertebrates. J. Anat. 199, 63-84.

Distel, H., and Ebbesson, S. O. (1981). Habenular projections in the monitor lizard (Varanus bengalensis). Exp. Brain Res. 43, 324-329.

Ellison, G. (1994). Stimulant-induced psychosis, the dopamine theory of schizophrenia, and the habenula. Brain Res. Brain Res. Rev. 19, 223-239.

Engbretson, G. A., Reiner, A., and Brecha, N. (1981). Habenular asymmetry and the central connections of the parietal eye of the lizard. J. Comp. Neurol. 198, 155-165.

Figdor, M. C., and Stern, C. D. (1993). Segmental organization of embryonic diencephalon. Nature 363, 630-634.

Finlay, B. L., and Darlington, R. B. (1995). Linked regularities in the development and evolution of mammalian brains. Science 268, 1578-1584.

Fowler, C. D., Lu, Q., Johnson, P. M., Marks, M. J., and Kenny, P. J. (2011). Habenular alpha5 nicotinic receptor subunit signalling controls nicotine intake. Nature 471, 597-601.

Funato, H., Saito-Nakazato, Y., and Takahashi, H. (2000). Axonal growth from the habenular nucleus along the neuromere boundary region of the diencephalon is regulated by semaphorin $3 \mathrm{~F}$ and netrin-1. Mol. Cell. Neurosci. 16, 206-220

Gamse, J. T., Kuan, Y. S., Macurak, M., Brosamle, C., Thisse, B., Thisse, C., and Halpern, M. E. (2005). Directional asymmetry of the zebrafish epithalamus guides dorsoventral innervation of the midbrain target. Development 132, 4869-4881.

Gamse, J. T., Thisse, C., Thisse, B., and Halpern, M. E. (2003). The parapineal mediates left-right asymmetry in the zebrafish diencephalon. Development 130, 1059-1068.

Goto, M., Swanson, L. W., and Canteras, N. S. (2001). Connections of the nucleus incertus. J. Comp. Neurol. 438, 86-122.

Guglielmotti, V., and Fiorino, L. (1998). Asymmetry in the left and right habenulo-interpeduncular tracts in the frog. Brain Res. Bull. 45, 105-110.

Hamada, H., Meno, C., Watanabe, D., and Saijoh, Y. (2002). Establishment of vertebrate left-right asymmetry. Nat. Rev. Genet. 3, 103-113.

Herkenham, M., and Nauta, W. J. (1979). Efferent connections of the habenular nuclei in the rat. J. Comp. Neurol. 187, 19-47.

Hikosaka, O. (2010). The habenula: from stress evasion to value-based decision-making. Nat. Rev. Neurosci. 11, 503-513.

Hirano, S., Yan, Q., and Suzuki, S. T. (1999). Expression of a novel protocadherin, OL-protocadherin, in a subset of functional systems of the developing mouse brain. J. Neurosci. 19, 995-1005.

Inbal, A., Kim, S. H., Shin, J., and Solnica-Krezel, L. (2007). Six3 represses nodal activity to establish early brain asymmetry in zebrafish. Neuron 55, 407-415.

Jhou, T. C., Geisler, S., Marinelli, M., Degarmo, B. A., and Zahm, D. S. (2009). The mesopontine rostromedial tegmental nucleus: A structure targeted by the lateral habenula that projects to the ventral tegmental area of Tsai and substantia nigra compacta. J. Comp. Neurol. 513, 566-596.

Kaufling, J., Veinante, P., Pawlowski, S. A., Freund-Mercier, M. J., and Barrot, M. (2009). Afferents to the GABAergic tail of the ventral tegmental area in the rat. J. Comp. Neurol. 513, 597-621.

Kemali, M., and Lazar, G. (1985). Cobalt injected into the right and left fasciculi retroflexes clarifies the organization of this pathway. J. Comp. Neurol. 233, 1-11.

Lecourtier, L., Neijt, H. C., and Kelly, P. H. (2004). Habenula lesions cause impaired cognitive performance in rats: implications for schizophrenia. Eur. J. Neurosci. 19, 2551-2560.

Li, B., Piriz, J., Mirrione, M., Chung, C., Proulx, C. D., Schulz, D., Henn, F., and Malinow, R. (2011). Synaptic potentiation onto habenula neurons in the learned helplessness model of depression. Nature 470, 535-539.

Liang, J. O., Etheridge, A., Hantsoo, L., Rubinstein, A. L., Nowak, S. J., Izpisua Belmonte, J. C., and Halpern, M. E. (2000). Asymmetric nodal signaling in the zebrafish diencephalon positions the pineal organ. Development 127, 5101-5112.

Long, S., Ahmad, N., and Rebagliati, M. (2003). The zebrafish nodalrelated gene southpaw is required for visceral and diencephalic leftright asymmetry. Development 130 , 2303-2316.

Louvi, A., and Artavanis-Tsakonas, S. (2006). Notch signalling in vertebrate neural development. Nat. Rev. Neurosci. 7, 93-102.

Martinez-Ferre, A., and Martinez, S. (2009). The development of the thalamic motor learning area is regulated by Fgf8 expression. J. Neurosci. 29, 13389-13400.

Matsumoto, M., and Hikosaka, O. (2007). Lateral habenula as a source of negative reward signals in dopamine neurons. Nature 447, 1111-1115.
Matsumoto, M., and Hikosaka, O. (2009). Representation of negative motivational value in the primate lateral habenula. Nat. Neurosci. 12, 77-84.

Mikula, S., Trotts, I., Stone, J. M., and Jones, E. G. (2007). Internet-enabled high-resolution brain mapping and virtual microscopy. Neuroimage 35, 9-15.

Morris, J. S., Smith, K. A., Cowen, P. J., Friston, K. J., and Dolan, R. J. (1999). Covariation of activity in habenula and dorsal raphe nuclei following tryptophan depletion. Neuroimage 10, 163-172.

Nishikawa, T., Fage, D., and Scatton, B. (1986). Evidence for, and nature of, the tonic inhibitory influence of habenulointerpeduncular pathways upon cerebral dopaminergic transmission in the rat. Brain Res. 373, 324-336.

Nishikawa, T., and Scatton, B. (1985). Inhibitory influence of GABA on central serotonergic transmission. Involvement of the habenulo-raphe pathways in the GABAergic inhibition of ascending cerebral serotonergic neurons. Brain Res. 331, 81-90.

Okamoto, H., Agetsuma, M., and Aizawa, H. (2011). Genetic dissection of the zebrafish habenula, a possible switching board for selection of behavioral strategy to cope with fear and anxiety. Dev. Neurobiol. doi: 10.1002/dneu.20913. [Epub ahead of print].

Olucha-Bordonau, F. E., Teruel, V., Barcia-Gonzalez, J., Ruiz-Torner, A., Valverde-Navarro, A. A., and Martinez-Soriano, F. (2003). Cytoarchitecture and efferent projections of the nucleus incertus of the rat. $J$. Comp. Neurol. 464, 62-97.

Quina, L. A., Wang, S., Ng, L., and Turner, E. E. (2009). Brn3a and Nurr1 mediate a gene regulatory pathway for habenula development. J. Neurosci. 29, 14309-14322.

Rebagliati, M. R., Toyama, R., Haffter, P., and Dawid, I. B. (1998). Cyclops encodes a nodal-related factor involved in midline signaling. Proc. Natl. Acad. Sci. U.S.A. 95, 9932-9937.

Regan, J. C., Concha, M. L., Roussigne, M., Russell, C., and Wilson, S. W. (2009). An Fgf8-dependent bistable cell migratory event establishes CNS asymmetry. Neuron 61, 27-34.

Roiser, J. P., Levy, J., Fromm, S. J., Nugent, A. C., Talagala, S. L., Hasler, G., Henn, F. A., Sahakian, B. J., and Drevets, W. C. (2009). The effects of tryptophan depletion on neural responses to emotional words in remitted depression. Biol. Psychiatry 66, 441-450.
Roussigne, M., Bianco, I. H., Wilson, S. W., and Blader, P. (2009). Nodal signalling imposes left-right asymmetry upon neurogenesis in the habenular nuclei. Development 136, 1549-1557.

Sahay, A., Molliver, M. E., Ginty, D. D., and Kolodkin, A. L. (2003). Semaphorin $3 \mathrm{~F}$ is critical for development of limbic system circuitry and is required in neurons for selective CNS axon guidance events. $J$. Neurosci. 23, 6671-6680.

Sampath, K., Rubinstein, A. L., Cheng, A. M., Liang, J. O., Fekany, K., Solnica-Krezel, L., Korzh, V., Halpern, M. E., and Wright, C. V. (1998). Induction of the zebrafish ventral brain and floorplate requires cyclops/nodal signalling. Nature 395, 185-189.

Sandyk, R. (1991). Relevance of the habenular complex to neuropsychiatry: a review and hypothesis. Int. J. Neurosci. 61, 189-219.

Sartorius, A., Kiening, K. L., Kirsch, P., Von Gall, C. C., Haberkorn, U., Unterberg, A. W., Henn, F. A., and Meyer-Lindenberg, A. (2010). Remission of major depression under deep brain stimulation of the lateral habenula in a therapyrefractory patient. Biol. Psychiatry 67, e9-e11.

Snelson, C. D., Santhakumar, K., Halpern, M. E., and Gamse, J. T. (2008). Tbx2b is required for the development of the parapineal organ. Development 135, 1693-1702.

Toga, A. W., and Thompson, P. M. (2003). Mapping brain asymmetry. Nat. Rev. Neurosci. 4, 37-48.

Tomizawa, K., Katayama, H., and Nakayasu, H. (2001). A novel monoclonal antibody recognizes a previously unknown subdivision of the habenulo-interpeduncular system in zebrafish. Brain Res. 901, 117-127.

Vallortigara, G., and Rogers, L. J. (2005). Survival with an asymmetrical brain: advantages and disadvantages of cerebral lateralization. Behav. Brain Sci. 28, 575-589.

Vue, T. Y., Aaker, J., Taniguchi, A., Kazemzadeh, C., Skidmore, J. M., Martin, D. M., Martin, J. F., Treier, M., and Nakagawa, Y. (2007). Characterization of progenitor domains in the developing mouse thalamus. J. Comp. Neurol. 505, 73-91.

Wang, R. Y., and Aghajanian, G. K. (1977). Physiological evidence for habenula as major link between forebrain and midbrain raphe. Science 197, 89-91.

Yanez, J., and Anadon, R. (1996). Afferent and efferent connections of 
the habenula in the rainbow trout (Oncorhynchus mykiss): an indocarbocyanine dye (DiI) study. J. Comp. Neurol. 372, 529-543.

Conflict of Interest Statement: The authors declare that the research was conducted in the absence of any commercial or financial relationships that could be construed as a potential conflict of interest.

Received: 01 November 2011; accepted: 01 December 2011; published online: 21 December 2011.
Citation: Aizawa H, Amo $R$ and Okamoto $H$ (2011) Phylogeny and ontogeny of the habenular structure. Front. Neurosci. 5:138. doi: 10.3389/fnins.2011.00138

This article was submitted to Frontiers in Neurogenesis, a specialty of Frontiers in Neuroscience.
Copyright (C) 2011 Aizawa, Amo and Okamoto. This is an open-access article distributed under the terms of the Creative Commons Attribution Non Commercial License, which permits noncommercial use, distribution, and reproduction in other forums, provided the original authors and source are credited. 